\title{
The magnetic structure of our Galaxy: a review of observations
}

\author{
JinLin Han \\ National Astronomical Observatories, Chinese Academy of Sciences \\ Jia-20 DaTun Road, Chaoyang District, Beijing 100012, China \\ email: hjl@bao.ac.cn
}

\begin{abstract}
The magnetic structure in the Galactic disk, the Galactic center and the Galactic halo can be delineated more clearly than ever before. In the Galactic disk, the magnetic structure has been revealed by starlight polarization within 2 or $3 \mathrm{kpc}$ of the Solar vicinity, by the distribution of the Zeeman splitting of $\mathrm{OH}$ masers in two or three nearby spiral arms, and by pulsar dispersion measures and rotation measures in nearly half of the disk. The polarized thermal dust emission of clouds at infrared, $\mathrm{mm}$ and submm wavelengths and the diffuse synchrotron emission are also related to the large-scale magnetic field in the disk. The rotation measures of extragalactic radio sources at low Galactic latitudes can be modeled by electron distributions and large-scale magnetic fields. The statistical properties of the magnetized interstellar medium at various scales have been studied using rotation measure data and polarization data. In the Galactic center, the non-thermal filaments indicate poloidal fields. There is no consensus on the field strength, maybe $\mathrm{mG}$, maybe tens of $\mu \mathrm{G}$. The polarized dust emission and much enhanced rotation measures of background radio sources are probably related to toroidal fields. In the Galactic halo, the antisymmetric RM sky reveals large-scale toroidal fields with reversed directions above and below the Galactic plane. Magnetic fields from all parts of our Galaxy are connected to form a global field structure. More observations are needed to explore the untouched regions and delineate how fields in different parts are connected.
\end{abstract}

Keywords. ISM: magnetic fields - Galaxy: structure - radio continuum: ISM

\section{Introduction}

Radio observations measure the total intensity, polarized intensity and rotation measure (RM). Sky maps of these quantities are all related to the magnetic fields in our Galaxy. Because the Milky Way is the largest edge-on galaxy in the sky, the synchrotron emission from relativistic electrons gyrating in the Galactic magnetic field is dominant in the radio sky. The stronger radio emission is observed nearer the Galactic plane, and strongest towards the Galactic central regions. The Galactic radio emission is polarized (Reich 2007), if it is not depolarized, best shown at tens of GHz (Page et al. 2007). The polarized emission undergoes Faraday rotation due to the Galactic magnetic fields and ionized electrons. The Faraday sky, i.e. the RM sky, is strikingly antisymmetric to the Galactic coordinate (Han et al. 1997, see Fig. 5).

The magnetic field of our Galaxy is more important in astrophysics and astroparticle physics than the fields in other galaxies. Magnetic fields are certainly one of key ingredients of the interstellar medium. Large-scale magnetic fields contribute to the hydrostatic balance and stability of the interstellar medium (Boulares \& Cox 1990), and even disk dynamics (Battaner \& Florido 2008). Magnetic fields in molecular clouds, which are closely related to the Galactic fields (Han \& Zhang 2007), play an important role in the star formation process (see a review by Heiles \& Crutcher 2005). More important is that the magnetic fields of our Galaxy are the main agent for transport of charged cosmic-rays 
(e.g. Tinyakov \& Tkachev 2002, Prouza \& Smída 2003). It is impossible to understand the origin and propagation of cosmic rays without adequate knowledge of Galactic magnetic fields. The Galactic radio emission and its polarization, which result from the Galactic magnetic fields, is found to heavily (up to $95 \%$ in polarization) "pollute" the measurements of the cosmological microwave background (CMB, e.g. Page et al. 2007). Galactic magnetic fields have suddenly become very important in the CMB studies of cosmology!

To understand magnetic fields, we have first to measure them and learn their properties. The galactic scale is in the middle between the stellar scale and the cosmological scale. It is on this scale that the magnetic fields can be well measured at present, at least from our Galaxy. Observations of galactic-scale magnetic fields provide the most important hints for and constraints on the origin and maintenance of the magnetic fields of Galactic objects and the fields in the universe. In the following, I will review the observed magnetic structures in the Galactic disk, the Galactic center and the Galactic halo.

\section{Magnetic fields in the Galactic disk}

Magnetic fields pervade the diffuse interstellar medium, molecular clouds, and very dense cloud cores or HII regions. When interstellar gas contracts to form a cloud or a cloud core the field is enhanced. Therefore, the observed field strength in clouds increases with gas density (Crutcher 1999). Here, I review the observational results of large-scale magnetic fields in the Galactic disk which are related to the diffuse medium and spiral structure and have a scale greater than $1 \mathrm{kpc}$. Magnetic fields on smaller scales will be mentioned only if they are related to the large-scale fields.

There are five observational tracers of the Galactic magnetic fields: polarization of starlight, polarized thermal dust emission from clouds, Zeeman splitting of lines, diffuse synchrotron radio emission, and Faraday rotation of polarized sources.

\section{Polarization of starlight}

Starlight becomes polarized when it passes through the interstellar medium and is absorbed or scattered by interstellar dust grains preferentially aligned by magnetic fields. The observed polarization is the integrated effect of scattering between the star and the Sun, and the "polarization vectors" show the averaged field orientation (weighted by the unknown local dust content). Starlight polarization data have been obtained for about 10,000 stars, mostly within 2 or $3 \mathrm{kpc}$ of the Sun (see Mathewson \& Ford 1970, Heiles 2000). Analysis of these data show that the local field is parallel to the Galactic plane and follows the local spiral arms (Heiles 1996). Starlight polarization data are difficult to use for detection of Galactic magnetic fields in a much larger region than 2 or $3 \mathrm{kpc}$. However, recent developments in instruments help to get a lot of new starlight polarization data for revealing magnetic fields in given objects (e.g. Feinstein et al. 2008).

\section{Polarized thermal dust emission from clouds}

In recent years, with development of instruments and backend technology (e.g. Hildebrand et al. 2000), observations of polarized thermal dust emission at mm, sub-mm and infrared bands have been used to detect the transverse orientation of magnetic fields in molecular clouds (see review by Heiles \& Crutcher 2005) on scales from 1 pc to tens of pc. The observed magnetic fields always have an hourglass shape, which indicates that the fields were enhanced when the clouds were formed by compressing the diffuse interstellar medium. Recently, Li et al. (2006) found that magnetic fields in molecular clouds seem to be preferentially parallel to the Galactic plane, indicating that the magnetic fields in the clouds preserve the fields frozen into the diffuse medium.

\section{Zeeman splitting of lines}

Zeeman splitting of spectral lines can measure in situ field strength of the line-of-sight component in molecular clouds or maser spots with a scale size $<1$ AU. To date, Zeeman 
splitting of emission or absorption lines has been detected from about 20 clouds (see a list in Crutcher 1999, Han \& Zhang 2007) and in OH masers associated with 140 HII or star-formation regions using single dishes (e.g. Caswell 2003) or interferometers (e.g. Fish et al. 2003). From collected Zeeman splitting data of $\mathrm{OH}$ masers of $\mathrm{HII}$ regions and $\mathrm{OH}$ or HI absorption or emission lines of molecular clouds themselves, Han \& Zhang (2007) found large-scale reversals in the sign of the line-of-sight component of the median field, indicating field reversals in a pattern similar to reversals revealed from pulsar RM data (see below). Evidently, magnetic fields on such a small scale are related to the large-scale Galactic magnetic fields to a very surprising extent (Reid \& Silverstein1990, Fish et al. 2003). Interstellar magnetic fields are apparently preserved as fossil fields through the cloud formation and star formation process, and even in stars (see G. Wade's talk in this volume). How can such coherent magnetic field directions be preserved from the low density medium $\left(\sim 1 \mathrm{~cm}^{-3}\right)$ to higher density clouds $\left(\sim 10^{3} \mathrm{~cm}^{-3}\right)$, even to the highest density in maser regions $\left(\sim 10^{7} \mathrm{~cm}^{-3}\right)$, with compression of 3 or even 10 orders of magnitude? It is a puzzle. The turbulent and violent processes in molecular clouds apparently cannot significantly alter the mean magnetic field.

\section{Diffuse synchrotron radio emission}

The total and polarized intensities of synchrotron emission are usually used to estimate the total and ordered field strength. For nearby galaxies, the strength of the regular largescale field is probably much overestimated from the polarization percentage, because the so-called ordered fields consist of the regular large-scale fields and anisotropic random fields (Han et al. 1999a) which both produce polarized emission.

We cannot have a face-on view of the global magnetic field structure in our Galaxy through polarized synchrotron emission, as is possible for nearby spiral galaxies (see R. Beck's talk in this volume). Polarization surveys of the Galactic plane have been comprehensively reviewed by Reich (2007). The observed polarized emission from the Galactic plane is the sum of all contributions with different polarization properties (i.e. polarization angles and polarization percentages) coming from various regions at different distances along a line of sight. Emission from more distant regions suffers from more Faraday effect produced by the foreground interstellar medium. Polarized emissions from different regions should "depolarize" each other when they are summed, which is more obvious at lower frequencies. Observations at higher frequencies (e.g. at $6 \mathrm{~cm}$ by Sun et al. 2007) show polarized structures at larger distances because depolarization is less severe. The polarized structures are closely related to the magnetic field structure where the emission is generated. Some large-angular-scale polarized features are seen emerging from the Galactic disk, for example, the North Polar Spur (e.g. Junkes et al. 1987).

\section{Faraday rotation of pulsars and radio sources}

Faraday rotation of linearly polarized radiation from pulsars and extragalactic radio sources (EGRs) is the most powerful probe of the diffuse magnetic field in the Galaxy (e.g. Han et al. 2006, Brown et al. 2007). Magnetic fields in a large part of the Galactic disk have been revealed by RM data of pulsars, which gives a measure of the line-of-sight component of the field. EGRs have the advantage of large numbers but pulsars have the advantage of being spread through the Galaxy at approximately known distances, allowing direct three-dimensional mapping of the magnetic fields. For a pulsar at distance $D$ (in pc), the RM (in radians $\mathrm{m}^{-2}$ ) is given by $\mathrm{RM}=0.810 \int_{0}^{D} n_{e} \mathbf{B} \cdot d \mathbf{l}$, where $n_{e}$ is the electron density in $\mathrm{cm}^{-3}, \mathbf{B}$ is the vector magnetic field in $\mu \mathrm{G}$ and $d \mathbf{l}$ is an elemental vector along the line of sight toward us (positive RMs correspond to fields directed toward us) in pc. With the pulsar dispersion measure, $\mathrm{DM}=\int_{0}^{D} n_{e} d l$, we obtain a direct estimate 


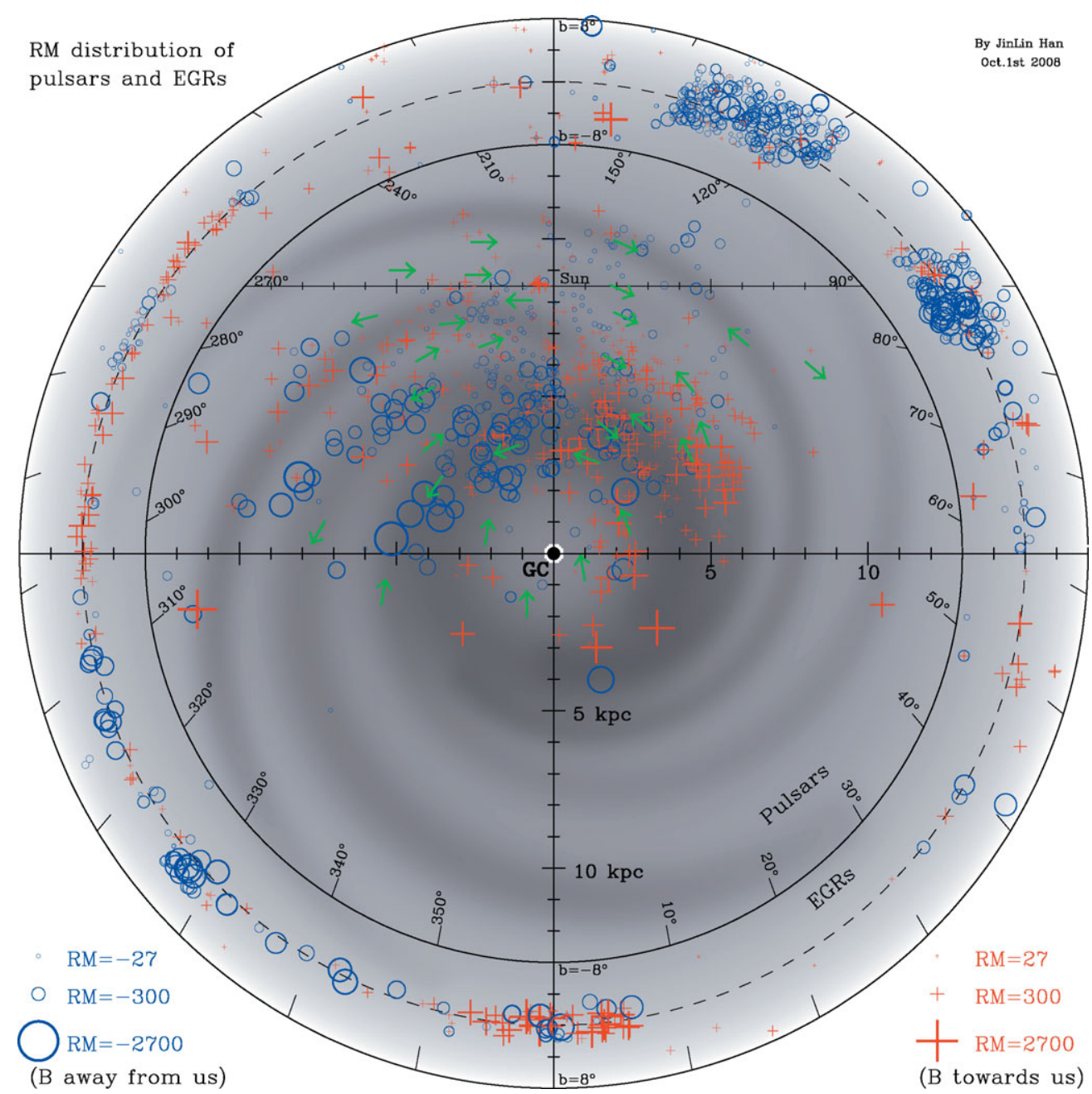

Figure 1. The RM distribution of 736 pulsars with $|b|<8^{\circ}$ projected onto the Galactic plane, including new data of Han et al. (2009, in preparation). The linear sizes of the symbols are proportional to the square root of the RM values with limits of \pm 27 and $\pm 2700 \mathrm{rad} \mathrm{m}^{-2}$. Positive RMs are shown by plus signs and negative RMs by open circles. The background shows the approximate locations of spiral arms used in the NE2001 electron density model (Cordes \& Lazio 2002). RMs of 1285 EGRs of $|b|<8^{\circ}$ (data mainly from Clegg et al. 1992, Gaensler et al. 2001, Brown et al. 2003, Roy et al. 2005, Brown et al. 2007 and other RM catalogs) are displayed in the outskirt ring according to their $l$ and $b$, with the same convention of RM symbols and limits. The large-scale structure of magnetic fields indicated by arrows was derived from pulsar RMs and comparison of them with RMs of background EGRs (details in Han et al. 2009). The averaged RM fluctuations with Galactic longitudes of EGRs are consistent with magnetic field directions derived from pulsar data, for example, in the 4th Galactic quadrant.

of the field strength weighted by the local free electron density

$$
\left\langle B_{\|}\right\rangle=\frac{\int_{0}^{D} n_{e} \mathbf{B} \cdot d \mathbf{l}}{\int_{0}^{D} n_{e} d l}=1.232 \frac{\mathrm{RM}}{\mathrm{DM}} .
$$

where RM and DM are in their usual units of $\operatorname{rad~m}^{-2}$ and $\mathrm{cm}^{-3} \mathrm{pc}$ and $B_{\|}$is in $\mu \mathrm{G}$.

Previous analyses of pulsar RM data have often used the model-fitting method (Manchester 1974, Thomson \& Nelson 1980, Rand \& Kulkarni 1989, Han \& Qiao 1994, Indrani 
\& Deshpande 1999, Noutsos et al. 2008), i.e., to model magnetic field structures in all of the paths from the pulsars to us (observer) and fit them, together with the electron density model, to the observed RM data. Significant improvement can be obtained when both RM and DM data are available for many pulsars in a given region with similar lines of sight. Measuring the gradient of RM with distance or DM is the most powerful method of determining both the direction and magnitude of the large-scale field local to that particular region of the Galaxy (Lyne \& Smith 1989, Han et al. 1999b, Han et al. 2002, Weisberg et al. 2004, Han et al. 2006). Field strengths in the region can be directly derived from the slope of trends in plots of RM versus DM. Based on Eq. (2.1), we get

$$
\left\langle B_{\|}\right\rangle_{d 1-d 0}=1.232 \frac{\Delta \mathrm{RM}}{\Delta \mathrm{DM}}
$$

where $\left\langle B_{||}\right\rangle_{d 1-d 0}$ is the mean line-of-sight field component in $\mu \mathrm{G}$ for the region between distances $d 0$ and $d 1, \Delta \mathrm{RM}=\mathrm{RM}_{d 1}-\mathrm{RM}_{d 0}$ and $\Delta \mathrm{DM}=\mathrm{DM}_{d 1}-\mathrm{DM}_{d 0}$.

i) Field structure

So far, RMs of 1021 pulsars have been observed (Hamilton \& Lyne 1987, Rand \& Lyne 1994, Qiao et al. 1995, Han et al. 1999b, Weisberg et al. 2004, Han et al. 2006, Noutsos et al. 2008), if new RMs of 477 pulsars by Han, van Straten, Manchester \& Demorest (2009, in preparation) are included (see Fig. 1). This enables us to investigate the structure of the Galactic magnetic field over a larger region than that previously possible. We have detected counterclockwise magnetic fields in the innermost arm, the Norma arm (Han et al. 2002). A more complete analysis for the fields of our Galaxy (Han et al. 2006 and Han et al. 2009, in preparation) from both RMs of pulsars and EGRs gives a picture for the coherent large-scale fields aligned with the spiral-arm structure, as shown in Fig.1: magnetic fields in all inner spiral arms are counterclockwise when viewed from the North Galactic pole. On the other hand, at least in the local region and in the inner Galaxy in the fourth quadrant, there is good evidence that the fields in interarm regions are similarly coherent, but reversed to be clockwise. There are at least four or five reversals in the fourth quadrant, probably occurring near the boundary of the spiral arms. In the Galactic central region interior to the Norma arm, new RM data of pulsars indicate that the fields are clockwise, reversed again from the counterclockwise field in the Norma arm. In the first Galactic quadrant, because the separations between spiral arms are so small, the RM data are dominated by counterclockwise fields in the arm regions though some (not many) negative pulsar RMs indicate clockwise fields in the interarm regions. The magnetic field in the Perseus arm cannot be determined well.

The averaged RM of EGRs in a given sky region reflect the common foreground Galactic RM contribution, which is the integration of $n_{e} B$ from the Sun to the outskirts of the Galactic disk. Comparison of the mean of RMs of background EGRs with RMs of foreground pulsars can reveal the magnetic fields behind the pulsars (see Fig. 1). However, the dominant contribution of RMs of EGRs behind the Galactic disk comes from the interstellar medium mostly in tangential regions. The fluctuations in the RM distribution of extragalactic radio sources (Clegg et al. 1992, Gaensler et al. 2001, Brown et al. 2003, Roy et al. 2005, Brown et al. 2007) with Galactic longitude, especially these of the fourth Galactic quadrant, are consistent with magnetic field directions derived from pulsar data (see Fig. 1). The negative RMs of EGRs in the 2nd quadrant suggest that the interarm fields both between the Sagittarius and Perseus arms and beyond the Perseus arm are predominantly clockwise.

Fitting various models to the new RM dataset shows different results (e.g. Noutsos et al. 2008). The most important is to have a model consistent with the detected field 
reversals. Looking at Table 2 of Men et al. (2008), one can see that any single-mode model is not enough to fit RM data though the bisymmetrical spiral model with field reversals is the best and gives the smallest $\chi^{2}$.

\section{ii) Field strength}

With much more pulsar RM data, for the first time, Han et al. (2006) were able to measure the regular field strength near the tangential regions in the 1st and 4th Galactic quadrants, and then plot the dependence of regular field strength as a function of galactocentric radius (Fig. 2). Although the "uncertainties", which in fact reflect the random fields, are large, the tendency is clear that fields get stronger at smaller Galactocentric radius and weaker in interarm regions. To parameterize the radial variation, an exponential function was used. This was chosen to give the smallest $\chi^{2}$ value and to avoid a singularity at $R=0$ (for $1 / R$ ) and unphysical values at large $\mathrm{R}$ (for the linear gradient). The function is, $B_{\text {reg }}(R)=B_{0} \exp \left[\frac{-\left(R-R_{\odot}\right)}{R_{\mathrm{B}}}\right]$, with the strength of the large-scale field at the Sun, $B_{0}=2.1 \pm 0.3 \mu \mathrm{G}$, and the scale radius $R_{\mathrm{B}}=8.5 \pm 4.7 \mathrm{kpc}$.

iii) Field statistics on small scales and spatial B-energy spectrum

Magnetic fields in our Galaxy exist on all scales. For the large-scale field, we can determine the field structure and field strength. To study small-scale magnetic fields, the only approach is to make statistics and have a description of their statistical properties.

Pulsar RMs have been used to study the small-scale random magnetic fields in the Galaxy. Some pairs of pulsars close in sky position have similar DMs but very different RMs, indicating an irregular field structure on scales of about 100 pc (Lyne \& Smith 1989). Some of these irregularities may result from HII regions in the line of sight to a pulsar (Mitra et al. 2003). It has been found from pulsar RMs that the random field has a strength of $B_{r} \sim 4-6 \mu \mathrm{G}$ independent of cell-size in the scale range of $10-100 \mathrm{pc}$ (Rand \& Kulkarni 1989, Ohno \& Shibata 1993). From pulsar RMs in a very large region of the Galactic disk, Han et al. (2004) obtained a power law distribution for magnetic field fluctuations of $E_{B}(k)=C\left(k / \mathrm{kpc}^{-1}\right)^{-0.37 \pm 0.10}$ at scales from $1 / k=0.5 \mathrm{kpc}$ to $15 \mathrm{kpc}$, with $C=(6.8 \pm 0.8) 10^{-13} \mathrm{erg}^{\mathrm{cm}^{-3}} \mathrm{kpc}$, corresponding to an rms field of $\sim 6 \mu \mathrm{G}$ in the scale range.

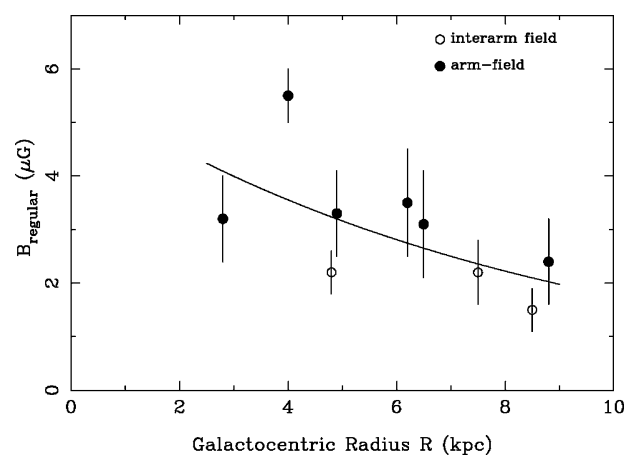

Figure 2. Variation of the large-scale regular field strength with Galactocentric radius derived from pulsar RM and DM data near the tangential regions (Han et al. 2006). Note that the "error-bars" are not caused by the uncertainty of the pulsar RM or DM data, but reflect the random magnetic fields in the regions.

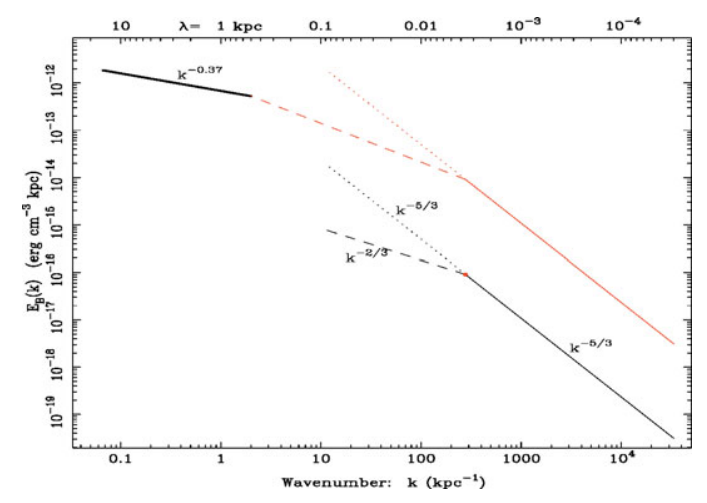

Figure 3. Composite spatial magnetic-energy spectrum in our Galaxy. See Han et al. (2004) for details. The thin solid and dashed/dotted lines at smaller scales are the Kolmogorov and 2D-turbulence spectra derived from Minter \& Spangler (1996), and the upper ones from new measurements of Minter (2004, private communication). 
Some results of magnetic field statistics were also obtained from RMs of EGRs. Armstrong et al. (1995) showed that the spatial power spectrum of electron density fluctuations from small scales up to a few pc could be approximated by a single power law with a 3D spectral index -3.7 , very close to the Kolmogorov spectrum. Magnetic fields on such small scales should follow the same power-law, since magnetic fields are frozen into the ionized interstellar medium. Minter \& Spangler (1996) found that structure functions of rotation measure and emission measure were consistent with a 3D-turbulence Kolmogorov spectrum of magnetic fields up to $4 \mathrm{pc}$, but with a $2 \mathrm{D}$ turbulence between $4 \mathrm{pc}$ and 80 pc. Haverkorn et al. (2006) found that the structure functions of RMs in the directions tangential to the arms have much larger slopes than those in the interarm directions, indicating that the arm regions are more turbulent. Sun \& Han (2004) found that the structure function for RMs at the two Galactic poles is flat, but at lower latitudes it becomes inclined with different slopes at different Galactic longitudes.

Combining the above information, one can get the spatial energy spectrum of Galactic magnetic fields (see Han et al. 2004) which should constrain the theoretical simulations (e.g. Balsara \& Kim 2005) of the generation and maintenance of Galactic magnetic fields. Evidently, on small scales, the distribution follows the Kolmogorov power-law spectrum, but at larger scales, it becomes flat and probably has two breaks at a scale of few pc and several tens of pc (Fig. 3). The interstellar magnetic fields probably become strongest at the scales of energy injection by supernova explosions and stellar winds (e.g. 1 to 10 pc).

\section{Magnetic fields in the Galactic center}

Within a few hundred pc of the Galactic center, both poloidal and toroidal magnetic fields have been observed. The non-thermal radio filaments (Fig. 4) discovered within $1^{\circ}$ from the Galactic center (Yusef-Zadeh et al. 1984, Lang et al. 1999, Yusef-Zadeh et al. 2004, Nord et al. 2004, LaRosa et al. 2004) indicate poloidal magnetic fields in the region within a few hundred parsecs of the center of the Galaxy. These filaments are highly polarized (Lang et al. 1999) and almost perpendicular to the Galactic plane, although some newly found examples are not so perpendicular to the Galactic plane (LaRosa et al. 2004). RM studies show that the magnetic field is aligned along the filaments (Lang et al. 1999). The filaments are probably illuminated flux tubes, with a field strength of about $1 \mathrm{mG}$ (Morris \& Serabyn 1996). LaRosa et al. (2005) detected diffuse radio emission of extent $400 \mathrm{pc}$ and on this basis argued for a weak pervasive field of tens of $\mu \mathrm{G}$ in the central region. However, this is the volume-averaged field strength in such a large region. The poloidal fields are possibly limited to a smaller central region. The newly discovered "double helix" nebula (Morris et al. 2006), with an estimated field strength of order $100 \mu \mathrm{G}$, reinforces the presence of strong poloidal magnetic fields in tube format merging from the rotating circumnuclear gas disk near the Galactic Center.

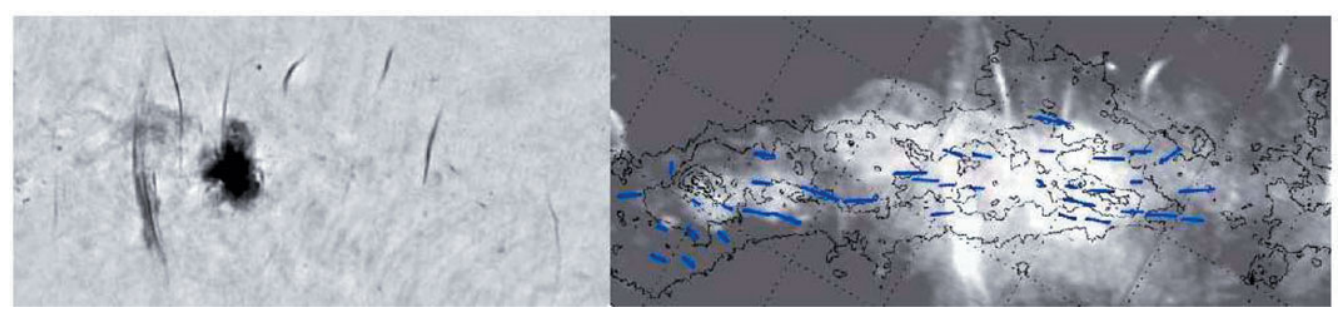

Figure 4. Non-thermal radio filaments discovered in the Galactic center $(\lambda 90 \mathrm{~cm}$ data from LaRosa et al. 2004) and the polarized thermal dust emission detected in the molecular cloud zone (bars in the right panel, see details in Novak et al. 2003). 
Polarized thermal dust emission has been detected in the molecular cloud zone at sub-mm wavelength (see Fig. 4, Novak et al. 2003, Chuss et al. 2003), which is probably related to the toroidal fields parallel to the Galactic plane and complements the poloidal fields shown by the vertical filaments. The observed molecular cloud zone has a size of a few hundred pc, and is possibly a ring-like cloud outside the central region with poloidal field (see Fig.1 of Chandran 2001). The sub-mm polarization observations of the cloud zone offer information only about the field orientations. Zeeman splitting measurements of HI absorption against Sgr A (e.g. Plante et al. 1995) or of the OH maser in the Sgr A region (Yusef-Zadeh et al. 1999) give a line-of-sight field strength of a few $\mathrm{mG}$ in the clouds. It is possible that toroidal fields in the clouds are sheared from the poloidal fields, so that the RM distribution of radio sources in this very central region could be antisymmetric (Novak et al. 2003).

Outside the central region of a few hundred pc to a few kpc, the structure in the stellar and gas distributions and the magnetic structure are all mysterious. There probably is a bar. The large-scale magnetic fields should be closely related to the material structure but have not been revealed yet. The large positive RMs of background radio sources within $|l|<6^{\circ}$ of the Galactic Center (Roy et al. 2005) are probably related to magnetic fields following the bar (Roy et al. 2008). Comparison of the RMs of these background radio sources with RMs of foreground pulsars (see Fig. 1) should be helpful in delineating the field structure.

\section{Magnetic fields in the Galactic halo}

Magnetic field structure in the Galactic halo can be revealed from RMs of EGRs if allowance can be made for sources with outstanding intrinsic RMs. The foreground Galactic RM is the common contribution to the observed RMs of all EGRs within a small patch of sky. After "anomalous" RMs are eliminated, the pattern for the Galactic RM can be obtained. Han et al. (1997) discarded any source if its RM deviated from the average RM of neighbouring sources by more than $3 \sigma$, and obtained a "cleaned" RM sky.

A striking antisymmetry in the inner Galaxy with respect to Galactic coordinates (see Fig. 5) has been identified from the cleaned RM sky (Han et al. 1997, Han et al. 1999b). The antisymmetry must result from azimuthal magnetic fields in the Galactic halo with reversed field directions below and above the Galactic plane. Such a field can be produced by an 'A0' dynamo mode. The observed filaments near the Galactic Center should result from the dipole field in this dynamo scenario. The local vertical field component of
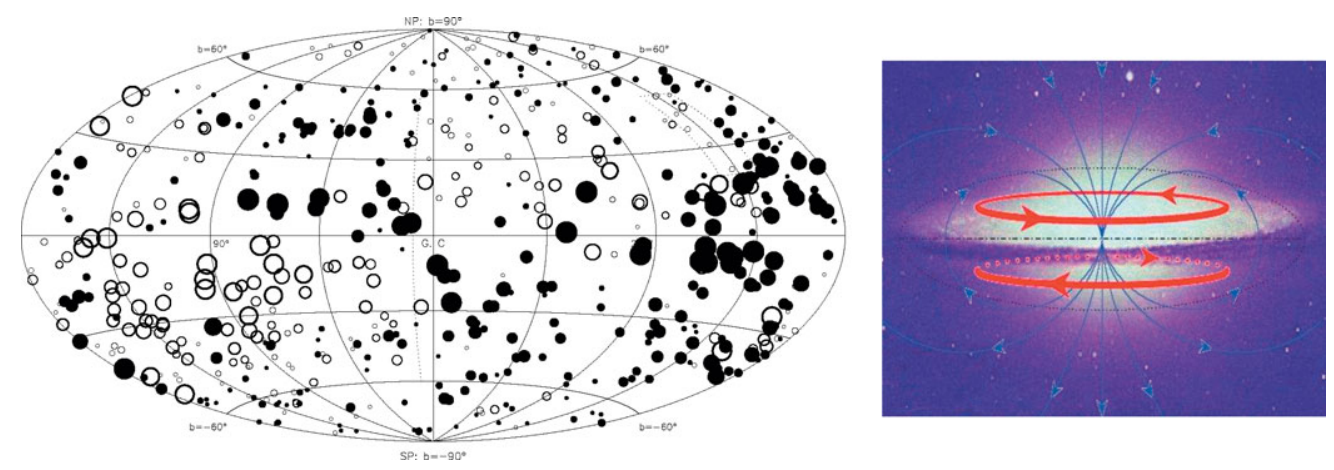

Figure 5. The antisymmetric rotation measure sky, derived from RMs of extragalactic radio sources after filtering out the outliers with anomalous RM values. The distribution corresponds to magnetic structure in the Galactic halo as illustrated on the right. See Han et al. $(1997,1999)$. 
$0.2 \mu \mathrm{G}$ (Han \& Qiao 1994, Han et al. 1999b) may be a part of this dipole field in the solar vicinity.

Han (2004) has shown that the RM amplitudes of extragalactic radio sources in the mid-latitudes of the inner Galaxy are systematically larger than those of pulsars, indicating that the antisymmetric magnetic fields are not local but are extended towards the Galactic center, far beyond the pulsars. Model-fitting by Sun et al. (2008) has confirmed this conclusion. The antisymmetry has been shown more clearly after adding newly observed RMs of more mid- or high latitude EGRs.

\section{Concluding remarks}

Only after the magnetic field, $\vec{B}(r, \theta, z)$, is known at all positions in our Galaxy will we have a complete picture of the magnetic structure of the Milky Way. With only a small number of measurements we must rely on fitting models to the data. "Partial" measurements in a greater number of regions, including quite large regions, are now available and we are able to "connect" the available measurements (pulsar RMs, maps of the RM sky) and outline some basic features of the Galactic magnetic field.

However, as seen from the above observational review, many regions of our Galaxy have not been observed very well for magnetic fields. For example, the farther half of the Galactic disk and the RM sky are not well known. Data in these regions are still scarce, either because of lack of field probes or limited capability of current instruments. In addition, when we try to get the large-scale field structure, the small-scale "random" fields, which are equally strong or even stronger, "interfere" with our measurements to a large extent. In the first Galactic quadrant, we obtained a large sample of pulsar RMs recently for the Galactic magnetic fields, but the spiral structure and pulsar distances are very uncertain. Statistical properties of fields on the energy-injection scales are not yet available, but are crucial for theoretical studies of magnetic field origin and maintenance. Magnetic fields in the disk-halo interface regions are certainly complex but few observations are available. To fully observe and understand the Galactic magnetic fields, there is a long way to go.

\section{Acknowledgements}

Thanks to Drs. Tom Landecker and A. Nelson for reading the manuscript. I am very grateful to colleagues who have collaborated with me for a long time on work on Galactic magnetic fields: Dr. R.N. Manchester from the Australia Telescope National Facility, CSIRO, Prof. G.J. Qiao from Peking University (China), Dr. Willem van Straten from Swinburne University (AU). The author is supported by the National Natural Science Foundation (NNSF) of China (10521001, 10773016 and 10833003) and the National Key Basic Research Science Foundation of China (2007CB815403).

\section{References}

Armstrong, J. W., Rickett, B. J., \& Spangler, S. R. 1995, ApJ 443, 209

Balsara, D. \& Kim, J. 2005, ApJ 634, 390

Battaner, E. \& Florido, E., 2007, AN 328, 92

Boulares, A. \& Cox, D. 1990, ApJ 365, 544

Brown, J. C., Taylor, A. R., \& Jackel, B. J. 2003, ApJS 145, 213

Brown, J. C., Haverkorn, M., Gaensler, B. M., et al. 2007, ApJ 663, 258

Caswell, J. L. 2003, MNRAS 341, 551

Chandran, B. D. G. 2001, ApJ 562, 737

Chuss, D. T., Davidson, J. A., Dotson, J. L., \& et al. 2003, ApJ 599, 1116

Clegg, A. W., Cordes, J. M., Simonetti, J. M., \& Kulkarni, S. R. 1992, ApJ 386, 143 
Cordes, J. M. \& Lazio, T. J. W. 2002, preprint (arXiv:astro-ph/0207156)

Crutcher, R. M. 1999, ApJ 520, 706

Feinstein, C., Vergne, M. M., Martńez, R., \& Orsatti, A. M., 2008, MNRAS 391, 447

Fish, V. L., Reid, M. J., Agron, A. L., \& Menten, K. M., 2003, ApJ 596, 328

Gaensler, B. M., Dickey, J. M., McClure-Griffiths, N. M., \& et al. 2001, ApJ 549, 959

Hamilton, P. A. \& Lyne, A. G. 1987, MNRAS 224, 1073

Han, J. L., 2004, In: The Magnetized Interstellar Medium, eds: Uyaniker, B., et al., GmbH, p. 3, Han, J. L. \& Qiao, G. J. 1994, A\&A 288, 759

Han, J. L., Beck, R., Ehle, M., Haynes, R. F., \& Wielebinski, R., 1999a, A\&A A 348, 405

Han, J. L., Ferriere, K., \& Manchester, R. N. 2004, ApJ 610, 820

Han, J. L., Manchester, R. N., Berkhuijsen, E. M., \& Beck, R. 1997, A\&\&A 322, 98

Han, J. L., Manchester, R. N., \& Qiao, G. J. 1999b, MNRAS 306, 371

Han, J. L., Manchester, R. N., Lyne, A. G., \& Qiao, G. J. 2002, ApJ 570, L17

Han, J. L., Manchester, R. N., Lyne, A. G., Qiao, G. J., \& van Straten, W. 2006, ApJ 642, 868.

Han, J. L., Zhang, J. S. 2007, A\&A 464, 609

Haverkorn, M., Gaensler, B. M., Brown, J. C., et al. 2006, ApJ 637, L33

Heiles, C. 1996, ApJ 462, 316

Heiles, C., 2000, AJ 119, 923

Heiles, C, \& Crutcher, R. 2005, In: Cosmic Magnetic Fields, LNP 664, 137

Hildebrand, R. H., Davidson, J. A., Dotson, J. L., et al., 2000, PASP 112, 1215

Indrani, C. \& Deshpande, A. A. 1999, New Astronomy 4, 33

Junkes, N., Fürst, E., \& Reich, W. 1987, A\&AS 69, 451

Lang, C. C., Morris, M., \& Echevarria, L. 1999, ApJ 526, 727

LaRosa, T. N., Brogan C. L., Shore S. N., et al. 2005, ApJ 626, L23

LaRosa, T. N., Nord, M. E., Lazio, T. J. W., \& Kassim, N. E. 2004, ApJ 607, 302

Li, H., Griffin, G. S., Krejny, M., \& et al. 2006, ApJ 648, 340

Lyne, A. G. \& Smith, F. G. 1989, MNRAS 237, 533

Manchester, R. N. 1974, ApJ 188, 637

Mathewson, D. S. \& Ford, V. L. 1970a, MemRAS 74, 139.

Men, H., Ferrière, K., \& Han, J. L. 2008, A\& A 486, 819

Minter, A. H. \& Spangler, S. R. 1996, ApJ 458, 194

Mitra, D., Wielebinski, R., Kramer, M., \& Jessner, A. 2003, A $\& A$ 398, 993

Morris, M., Serabyn, 1996, ARA\&A 34, 645

Morris, M., Uchida, K.,\& Do, T. 2006, Nature 440, 308

Nord, M. E., Lazio, T. J. W., Kassim, N. E., et al. 2004, AJ 128, 1646

Novak, G., Chuss, D. T., Renbarger, T., \& et al. 2003, ApJ 583, L83

Noutsos, A., Johnston, S., Kramer, M., \& Karastergiou, A. 2008, MNRAS 386, 1881

Ohno, H., Shibata, S., 1993, MNRAS 262, 953

Page, L., et al., 2007, ApJS 170, 335

Plante, R.. L., Lo, K. Y., \& Crutcher, R.. M., 1995, ApJ 445, L113

Prouza, M. \& Smída, R. 2003, A $\& A$ 410, 1

Qiao, G. J., Manchester, R. N., Lyne, A. G., \& Gould, D. M. 1995, MNRAS 274, 572

Rand, R. J. \& Kulkarni, S. R. 1989, ApJ 343, 760

Rand, R. J. \& Lyne, A. G. 1994, MNRAS 268, 497

Reich, W. 2007, in: Cosmic Polarization, (astro-ph/0603465), p.91

Reid, M. J. \& Silverstein, E. M., 1990, ApJ 361, 483

Roy, S., Rao, A. P., \& Subrahmanyan, R. 2005, MNRAS 360, 1305

Roy, S., Rao, A. P., \& Subrahmanyan, R. 2008, A\& A 478, 435

Sun, X. H. \& Han, J. L. 2004, in: Magnetized Interstellar Medium, eds: Uyaniker, B., et al., GmbH, p. 25

Sun, X.H., Han, J.L., Reich, W., \& et al. 2007, A 6 A 463, 993

Sun, X. H., Reich, W., Waelkens, A., \& Enßlin, T. A. 2008, A\& A 477, 573

Thomson, R. C. \& Nelson, A. H. 1980, MNRAS 191, 863

Tinyakov, P. G. \& Tkachev, I. I. 2002, Astroparticle Physics 18, 165

Weisberg, J. M., Cordes, J. M., Kuan, et al. 2004, ApJS 150, 317

Yusef-Zadeh, F., Hewitt, J. W., \& Cotton, W. A. 2004, ApJS 155, 421

Yusef-Zadeh, F., Morris, M., \& Chance, D. 1984, Nature 310, 557

Yusef-Zadeh, F., Roberts, D. A., Goss, W. M., et al. 1999, ApJ 512, 230 


\section{Discussion}

NELSON: Can we obtain any information on the field strength in the outer disk of our Galaxy from the extragalactic rotation measures, say from $20-30 \mathrm{kpc}$ radius?

HAN: Hard. From pulsar RMs, we can determine the field strength up to $R \sim 10 \mathrm{kpc}$. For the outer Galaxy, the RMs of extragalactic radio sources have to be fitted with a model of $B$ and a model of $n_{e}$. Given the many uncertainties in these models for the outer disk, it is really hard to determine the field strength. Nevertheless, from the determined variation of the field strength with the Galactocentric radius, you can extrapolate the field strength to the outer Galaxy.

De Gouveia Dal Pino: Do you see any indication of an X-shaped magnetic structure similar to the one we see in other edge-on star-forming galaxies?

HAN: For this purpose, one needs to map the polarization in a large field-of-view around the Galactic center. At present, the available good maps with a field-of-view of 1 or 2 degrees were made with the VLA, and show the non-thermal filaments indicating the poloidal fields. It is impossible to restore the absolute level of $U$ and $Q$ in these maps. Therefore we can not map it now even if there may be such an X-shaped field structure. It is possible that the double helix nebula seen by Morris et al. (2006) and the filaments observed in the Galactic center region are somehow the foot or leg part of such a structure.

LANDECKER: I am concerned that the interpretation of RMs of pulsar and extragalactic radio sources is heavily dependent on the models of electron density distribution of Taylor \& Cordes (1993) or Cordes \& Lazio (2002). These models incorporate implicitly a spiral pattern for the Galaxy, going back to the work of Georgelin \& Georgelin (1976) whose basic assumption is that there is a neat spiral pattern in the structure of the Milky Way. Please comment.

HAN: You are right on the electron density model. For interpretation for pulsar RMs, we work on the RM variations against not only the pulsar distances but also the observed DM. Considering that the relative positions of pulsars are more closely related to the DM rather than the electron density model, we believe that the magnetic structure derived from pulsar RMs and DMs are less model-dependent. For interpretation for RMs of extragalactic radio sources, yes, it is very heavily dependent on the electron density model and non "DM" to use.

From all observations available, evidence for spiral arms is so strong, therefore it is a correct idea to incorporate the spiral structure into any electron density model. Now, the spiral arms in the fourth Galactic quadrant have been better determined, while in the first quadrant, it is much less clear. Also, the electron density within a few kpc from the Galactic center is not clear at all. The electron density model and hence the pulsar distances therefore have a large uncertainty in these regions. Future electron density models will be probably improved there. 


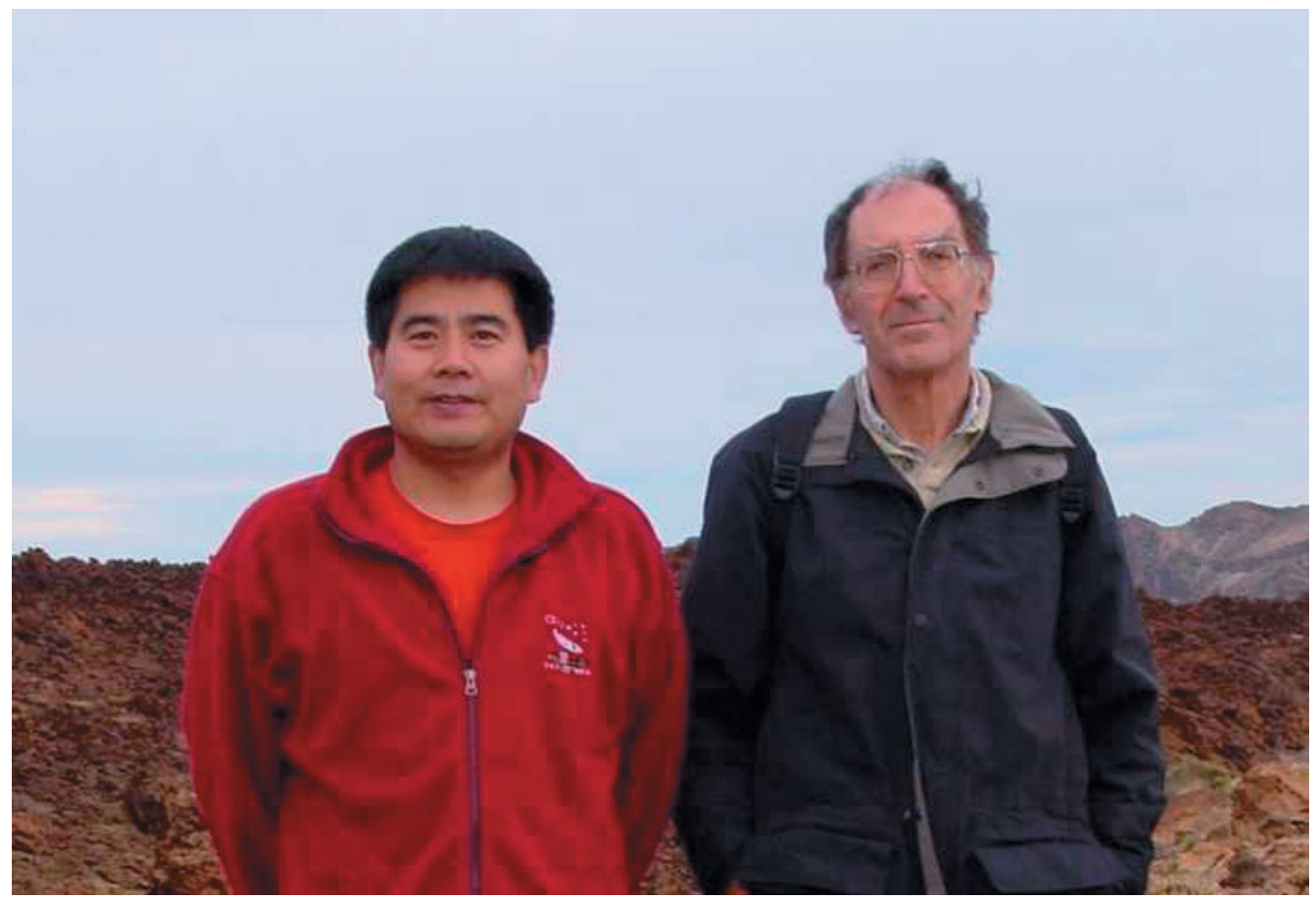

JinLin Han (left), and Tom Landecker

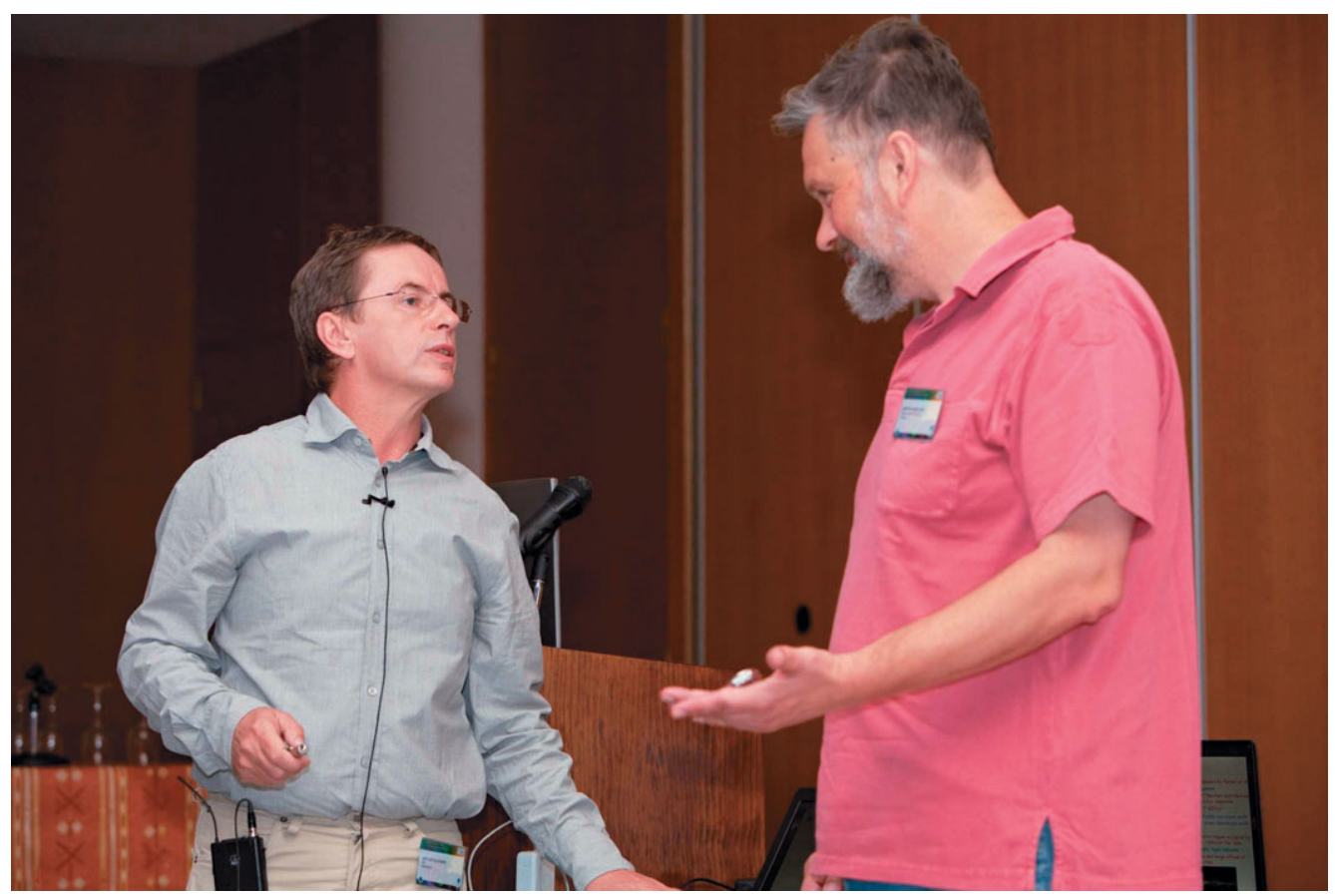

Detlef Elstner (left), arguing with the session chairman Dimitry Sokoloff 